\title{
Ein Schlüssel zu grünem Wasserstoff
}

\author{
Eine Schicht, dünn wie ein einziges Atom, macht einen gewaltigen Unterschied bei der \\ Wasserstoffproduktion: Auf der Oberfläche einer Elektrode verdoppelt sie die Menge des \\ Wassers, das in einer Elektrolyse-Anlage gespalten wird - ohne dass sich dabei der \\ Energiebedarf erhöht. Damit verdoppelt die ultradünne Schicht auch die Menge des \\ produzierten Wasserstoffs, ohne dass die Kosten steigen.
}

Forscher aus Jülich, Aachen, Stanford und Berkeley haben den schichtweisen Aufbau eines keramischen Katalysatormaterials untersucht und herausgefunden, dass eine atomar dünne Oberflächenschicht die Aktivität für die Wasserspaltungsreaktion verdoppeln kann. Damit verdoppelt die ultradünne Schicht auch die Menge des produzierten Wasserstoffs, ohne dass die Kosten steigen. „Uns ist es gelungen, durch Untersuchung eines modellhaften Materials ein detaillierteres Verständnis davon zu gewinnen, wie sich die Eigenschaften einer katalytisch wirkenden Elektrode aus ihrem Aufbau ergeben", sagt Christoph Bäumer, Erstautor der Studie. Gefördert mit einem, Global Fellowship der Marie-Skłodowska-Curie-Maßnahmen hat er die Forschung sowohl in Jülich und Aachen als auch in den USA vorangetrieben. Der Materialwissenschaftler weiter: „Von diesem erweiterten Verständnis erhoffen wir uns, dass in Zukunft bessere Katalysatoren entwickelt werden können, die grünen Wasserstoff energieeffizienter und damit kostengünstiger herstellen als bisher."

\section{Wann ist Wasserstoff grün?}

Als grün wird das farblose Wasserstoff-Gas dann bezeichnet, wenn es durch die Elektrolyse von Wasser klimaneutral mit Strom aus erneuerbaren Quellen gewonnen wird. Wasserstoff gilt als wesentlicher Baustein der Energiewende, unter anderem, weil er Windund Sonnenenergie in Zeiten des Überangebots speichern und später wieder freisetzen kann. Die elektrolytische Wasserstoffproduktion an der negativ geladenen Elektrode (Kathode) kann allerdings nicht ohne eine Sauerstoffentwicklung an der positiv geladenen Elektrode (Anode) stattfinden. Katalysatoren, die diese Sauerstoffentwicklung begünstigen, machen somit auch den Gesamtprozess energieeffizienter. Der hohe Energiebedarf ist bisher eine der Haupthürden für die breite Nutzung von Wasserstoff.

Lanthannickelat mit der Summenformel $\mathrm{LaNiO}_{3}$, zur Materialklasse der Perowskite gehörend, ist ein solcher Katalysator. In seiner Kristallstruktur wechseln sich Nickel-Oxidund Lanthan-Oxid-Schichten ab. „Wir haben Lanthannickelat-Katalysatoren präziser produziert und genauer untersucht als andere Wissenschaftler vorher", sagt Felix Gunkel vom Peter Grünberg Institut, der die Forschungsaktivitäten in Jülich geleitet hat. Denn die Forschenden stellten zwei verschiedene Arten hochreiner $\mathrm{LaNiO}_{3}$-Kristalle her: Bei der einen Art enden die Kristalle an einer Oberfläche, in der sich nur Lanthan- und Sauerstoff-Atome befinden. Fachleute sprechen von einer Lanthan-Terminierung. Bei der anderen Art bilden Nickel- und SauerstoffAtome die Oberfläche (Nickel-Terminierung) (Bild 1).

\section{Besonders aktive Katalysatoren}

Es zeigte sich, dass eine Nickel-terminierte Anode in der gleichen Zeit doppelt so viel Sauerstoff produziert wie eine ebenso große Lanthan-terminierte Elektrode. „Überraschenderweise ist also eine einzige Lage von Nickel- und Sauerstoffatomen für eine ganzerhebliche Steigerung der katalytischen Aktivität des Materials verantwortlich", so Bäumer. Das Wissenschaftlerteam konnte auch einen Grund dafür finden: Während der Elektrolyse entsteht auf dem Nickel-terminierten Kristall eine ungeordnete, katalytisch sehr aktive Schicht aus Nickeldioxid, die sich bei Lanthan-Terminierung nicht bilden kann. Diese neu gebildete Nickeloxidschicht hat im Vergleich zur Ausgangsstruktur ideale Bindungszustände zwi-

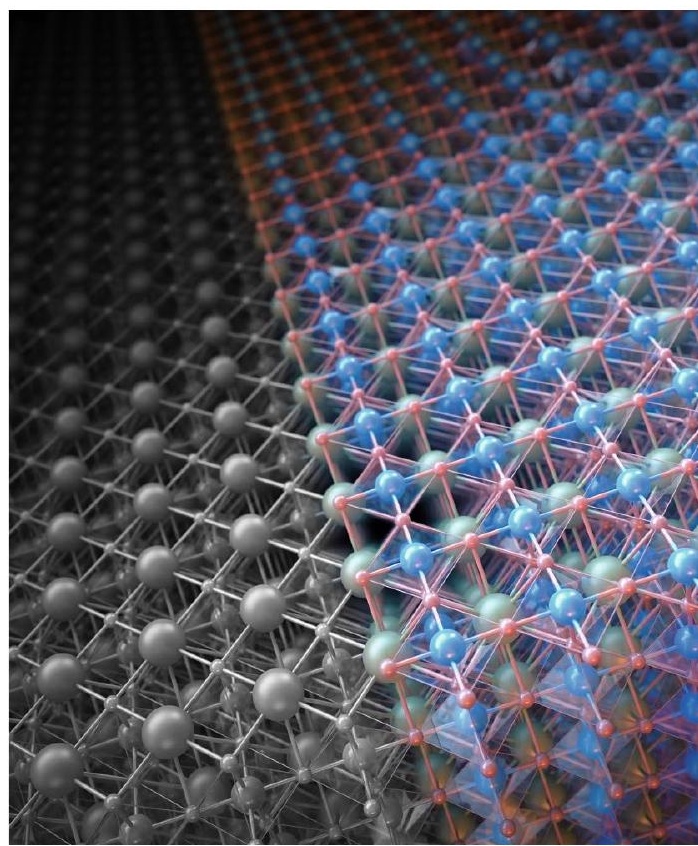

Bild 1 Zwei mögliche Oberflächenschichten für ein Katalysatormaterial: Die graue Oberfläche ist Lanthanoxid. Die bunte Oberfläche ist Nickeloxid, das durch eine Umlagerung seiner Atome während der Reaktion doppelt so aktiv ist wie die Lanthanoxidoberfläche. Die Lanthanatome sind in grün, Nickel in blau und Sauerstoff in rot abgebildet. (@ CUBE3D Graphic)

schen Nickelionen und Sauerstoff- oder Hydroxidionen, was die Aktivität erhöht. „Unsere Ergebnisse weisen darauf hin, dass man auch bei anderen Materialien die Terminierung und die daraus folgende Strukturänderung unter Betriebsbedingungen berücksichtigen muss, wenn man besonders aktive Katalysatoren entwickeln will", sagt William Chueh, Professor an der Stanford University. 4

Kontakt:

Forschungszentrum Jülich, Peter Grünberg Institut, Bereich Elektronische Materialien (PGI-7), www.fz-juelich.de/pgi/pgi-7 